\title{
Análise dos modelos de comportamento de busca e uso de informação nas dissertações e teses dos PPGCl: Uma proposta de ampliação ao modelo de Ellis
}

\author{
Hamilton Rodrigues Tabosa * \\ Virginia Bentes Pinto
}

Artículo recibido:

28 de agosto de 2013.

Artículo aceptado:

9 de septiembre de 2013.

\section{Resumen}

Análisis de los modelos de comportamiento de búsqueda y uso de la información en los ensayos y tesis del PPGCI: una propuesta de ampliación al modelo de Ellis. El objetivo del presente trabajo es analizar cómo los estudios e investigaciones sobre el comportamiento de búsqueda y uso de la información se están diseñando en ensayos y tesis en los programas de posgrado en Ciencias de la Información en Brasil durante el periodo 20002012, con el fin de expandir el modelo de Ellis. Se parte del estudio del modelo de comportamiento en la búsqueda y uso de la información de Ellis, Cox y Ha11 (1993), teniendo en cuenta la propuesta de Crespo (2005), para proponer una ampliación del modelo de

* Los dos autores pertenecen a la Universidad Federal de Ceará (UFC), Brasil. (Hamilton: hrtabosa@gmail.com); (Virginia: bentespinto@yahoo.com.br). 
comportamiento de la búsqueda y uso de información de Ellis (1989). La metodología del estudio empírico consistió en la investigación bibliográfica con enfoque cualitativo, para ello se analizaron 26 informes de investigación (22 ensayos y cuatro tesis) en el ámbito de los estudios de uso y usuarios de la información. Como resultado del análisis del corpus documental se confirmó la necesidad de una mayor expansión del modelo de comportamiento de búsqueda y uso de información de Ellis, añadiendo la categoría Compartir.

Palabras clave: Comportamiento de los usuarios; Modelos de comportamiento de búsqueda; Búsqueda de información.

\section{Abstract}

Analysis of information use and search behavior models in dissertations and theses of PPGCI: A proposal to broaden in the Ellis Model

Hamilton Rodrigues-Tabosa and Virginia Bentes-Pinto

With a view toward modifying the Ellis model, this study examines how search behavior and information use studies are employed in dissertations and theses defended in Brazilian Information Science postgraduate programs in the period 2000-2012. In light of the proposition offered by Crespo (2005), researchers employed the search behavior and information use model developed by Ellis, Cox and Hall (1993) in order to propose an addition to the Ellis (1989) model. The methodology of the empirical study entailed a qualitative review of twenty-six research reports, comprised of twenty-four theses and two essays. This review confirmed the need for the addition of Sharing as a category.

Keywords: User Behavior; Information Seeking Behavior Models; Information Search.

\section{INTRODUÇÃO}

a atualidade, diversos fatores tecnológicos e sociais estão influenciando
no modo como nos relacionamos, comunicamos, trabalhamos, produ- 
zimos conhecimento e, inevitavelmente, no modo como os registramos e os acessamos. Desde a segunda metade do século XX, a Ciência da Informação (CI) tem demonstrado interesse em conhecer a estrutura bem como as características que envolvem a interação do sujeito com os recursos, serviços e fontes de informação e pôs-se a desenvolver estudos de comportamento de busca e uso da informação, ou seja, estudos sobre os esquemas de ações que um indivíduo realiza para satisfazer suas necessidades informacionais, para tanto, são realizados estudos e pesquisas sobre os comportamentos de busca e uso da informação, em espaços informacionais analógicos e digitais.

Embora Wilson (2000) afirme que o marco inicial das pesquisas sobre o comportamento de busca e uso da informação tenha sido a realização da Royal Society Scientific Information Conference em 1948, Pinheiro (1982) afirma que o Centre for Research on User Studies (CRUS), em Sheffield-Inglaterra, mapeou estudos referentes a esse tema, desde o período de 1919-1979, e que eles se intensificaram a partir das décadas de sessenta e setenta. No entanto, foi nas décadas de oitenta e noventa que surgiram proposições de alguns modelos ou padrões de comportamento de busca e uso de informação, tais como os de Wilson (1981), Dervin (1983), Ellis (1989) ampliado por Ellis, Cox e Hall (1993), Kuhlthau (1991) e Wilson e Walsh (1996). Esses modelos ou padrões se referem a uma previsibilidade no que se refere às decisões e às ações tomadas pelos sujeitos quando diante de uma necessidade de informação, levando-os a buscar a satisfação dessa necessidade para a solução de um problema.

Naturalmente, conforme a sociedade se torna cada vez mais tecnológica e, considerando que o modo como se acessa informação nos dias de hoje difere do que ocorria na década de 80, é primordial que a CI continue investigando como as pessoas se comportam para resolver problemas informacionais com a finalidade de melhor oferecer produtos e serviços à sociedade. Também devemos considerar que as pessoas na sua individualidade, bem como as coletividades, são subjetivas, exercem e ao mesmo tempo sofrem a ação do social, coerções que interferem em seu comportamento dentro dos grupos sociais. Assim, as sociedades não são estáticas, elas mudam conforme o tempo, o que se reflete nos comportamentos dos grupos sociais que a compõem, motivo pelo qual os estudos e pesquisas referentes ao comportamento de busca e uso de informação devem ser atualizados, para se adequar à realidade sócio-temporal e mesmo cognitiva dos sujeitos.

Além disso, é preciso se ter consciência de que jamais teremos um modelo que represente, inequivocamente e de modo engessado, as necessidades e usos de informação dos sujeitos, pois eles são dotados de ações cognitivas e, portanto, dinâmicos e não haverá qualquer possibilidade de que sejam mol- 
dados em suas buscas, mesmo que os sistemas tentem fazê-lo. A etnografia do comportamento de buscas e usos de informação é impar a cada indivíduo. Isso se exemplifica pelas ampliações do modelo de Ellis (1989) proposta por Ellis, Cox e Hall em (1993) e por Crespo (2005).

Neste artigo, partimos do estudo do modelo de comportamento de busca e uso de informação de Ellis, Cox e Hall (1993), considerando a proposição de Crespo (2005) e de Barros (2008), para também propormos uma ampliação a esse modelo.

Sendo assim, o problema desta pesquisa contempla a seguinte questão: de que modo estão sendo pensados os estudos e pesquisas sobre comportamentos de busca e uso de informação nas dissertações e teses defendidas nos programas de pós-graduação em Ciência da Informação no período de 2000 a 2012?

Visando encontrar respostas para esse questionamento, definimos como objetivo básico desta pesquisa: analisar o modo como os estudos e pesquisas sobre comportamentos de busca e uso de informação estão sendo pensados nas dissertações e teses defendidas nos programas de pós-graduação em Ciência da Informação defendidas no período de 12 anos, com vistas à ampliação ao modelo de Ellis.

A metodologia do estudo empírico foi realizada por meio da pesquisa bibliográfica com enfoque qualitativo, tendo sido analisados vinte e seis relatórios de pesquisa: vinte e duas dissertações e quatro teses (dentro do escopo dos estudos de uso e usuários da informação), defendidas em programas de pós-graduação em CI no Brasil de 2000 a 2012, por meio de consultas à Biblioteca Digital Brasileira de Teses e Dissertações (BDTD) ${ }^{1}$ e nos repositórios institucionais das universidades onde funcionam cursos de pós-graduação em nível de mestrado e/ou doutorado.

O corpus documental para o estudo foi selecionado por meio da utilização do campo de busca avançada presente nas interfaces dos bancos de dados, e que respondeu a estratégias de buscas com os operadores booleanos "e" e "ou", ou equivalentes, empregados para ligar os termos "necessidade", "informação", "uso", "usuários" e "comportamento", que aparecessem no resumo das dissertações ou teses, para identificar pesquisas que tivessem como temática principal os estudos sobre comportamentos de busca e uso da informação.

Foi criada uma planilha para consolidar os dados e facilitar a leitura tendo por base as categorias: autor, tipo de trabalho (dissertação ou tese), objetivo geral, metodologia e resultados. O objetivo geral de cada trabalho foi transcrito fielmente na coluna correspondente, porém, na coluna que coube 
à determinação da metodologia, foram usados termos e expressões resumidas para indicar a escolha metodológica do pesquisador, com relação ao método, tipo de pesquisa quanto aos objetivos (exploratória, descritiva, dentre outros), natureza (qualitativa, quantitativa, etc.), técnica de coleta de dados (entrevistas, questionários e sua tipologia, etc.), técnica de análise de dados (análise do discurso, análise de conteúdo, etc.), e assim por diante. Também foi feita a transcrição de trechos retirados do resumo e/ou do capítulo destinado à análise dos dados e/ou da conclusão de cada trabalho, para identificar os principais resultados alcançados por cada pesquisa, no intuito de se traçar um panorama dos resultados alcançados e identificar regularidades nos comportamentos de busca e uso da informação dos sujeitos de cada pesquisa, que pudessem indicar a necessidade de ampliação/atualização do modelo de Ellis.

Como resultado da análise do nosso corpus documental, confirmamos a necessidade de mais uma ampliação do modelo de comportamento de busca e uso da informação de Ellis, sobre a qual falaremos adiante.

\section{Estudos DE COMPORTAMENTO DE BUSCA E USO DE INFORMAÇÃO}

Estudar comportamentos de busca e uso de informação implica adentrar no contexto interdisciplinar, principalmente com a Biologia, a Antropologia, a Sociobiologia e a Psicologia Evolucionista, entre outras, a fim de compreender tal processo. Os estudos de Laland e Brown (2002) trazem uma reflexão bastante interessante sobre as mudanças de paradigma sobre o comportamento humano. Porém, para a análise proposta neste artigo não nos deteremos nessas teorias. Entretanto, entendemos que os estudos de comportamentos de usuários não podem ser vistos sem que se tenha em conta que o ato de busca e usos de informação configuram-se como ações relativas a situações, logo, podem ser considerados dentro dos estudos de situação de anomalia ou estado de anomalia do conhecimento, como defende Belkin (1980) no seu modelo Anomalous State of Knowledge (ASK).

Mesquita e Duarte (1996) definem comportamento ou conduta como o termo que designa a atividade global ou conjunto dos atos de um indivíduo perante uma situação ou conjunto de estímulos, ou seja, é a resposta que um organismo dá, ou a sua reação, perante a situação que a suscita. Podemos, então, considerar comportamento de busca e uso de informação como o conjunto de ações de um sujeito (usuário de informação) diante de uma necessidade de informação (estímulo), ou seja, o caminho que ele percorre para buscar, acessar e usar informação para satisfazer sua necessidade. 
Os estudos de comportamento do usuário abrangem vários aspectos, conforme Crespo (2005): a identificação das necessidades de informação, o modo como os indivíduos usam a informação, os fatores internos e externos que influenciam o processo, entre outros.

Consideramos oportuno esclarecer os conceitos que adotamos para alguns termos empregados neste artigo, conforme Wilson (2000):

a) comportamento informacional é a totalidade de comportamentos humanos em relação às fontes e canais de informação, incluindo as ações que o usuário delibera tomar para alcançar seu objetivo e as ações em que ele não tem, necessariamente, a intenção de obter informação, por exemplo: ouvir, despropositadamente, a conversa de outras pessoas, rádio ou TV;

b) comportamento de busca da informação parte de uma necessidade e constitui-se na busca proposital por informação para resolver um problema ou realizar uma tarefa. Durante uma busca, o usuário pode utilizar recursos manuais e/ou eletrônicos.

c) comportamento de uso da informação consiste nas atividades físicas e mentais que envolvem a assimilação do conhecimento e sua incorporação cognitiva por parte do usuário;

Wilson (2000) citado por Cruz (2011) argumenta que os estudos de usuários estão inexoravelmente ligados à definição da CI, compreendida como uma ciência que estuda, dentre outras problemáticas, como os usuários se comportam, como traçam caminhos particulares para obter a informação da qual necessitam. Segundo o autor, a CI sempre tem se preocupado com o estudo do usuário da informação, desde seu alvorecer.

Durante o período em que a CI vivia o paradigma positivista (Capurro, 2007), os estudos com foco no usuário se davam por meio de medições de frequência à biblioteca, número de empréstimos, dentre outras estatísticas e contabilizações inadequadas ao estudo das subjetividades inerentes ao comportamento humano. Posteriormente, desenvolveram-se outros estudos com base no cognitivismo, que procuraram identificar sentimentos e motivações dos sujeitos na busca por informação, destacando-se os estudos de Belkin (1980), principalmente.

Segundo Wilson (1981), o estudo do comportamento de busca de informação presume a existência de uma necessidade de informação, ou seja, o sujeito não se sente satisfeito com a informação que possui e busca meios de sanar essa necessidade. No entanto, o autor alerta para o fato de que nem sempre a busca irá efetivamente acontecer e que ela pode também ser inter- 
rompida. Isso porque existem fatores externos ao sujeito que interferem no processo, ao que ele chama de barreiras, que podem ser pessoais, interpessoais e ambientais.

É importante frisar que os estudos de comportamentos de busca se desenvolvem sempre dentro de um grupo ou comunidade e que os resultados desses estudos não descrevem, necessariamente, um comportamento universal. Sempre haverá desvios ao modelo ou padrão, movidos pelas especificidades do grupo com qual o estudo foi realizado, muito embora possam ser traçadas regularidades presentes em todos os grupos.

Atualmente, com a popularização da internet, muitas vezes o próprio usuário utiliza a web para procurar a informação de que necessita, tornando-se mais independente e indo menos à biblioteca (Milne, 1999), utilizando outras fontes e se comportando de maneira diferente. Essa realidade demanda do profissional da informação novos estudos para melhor caracterizar e compreender o novo perfil do usuário da informação e as implicações dessas mudanças.

\section{Modelo de Comportamento de Busca e Uso de Informação de Ellis}

Ellis (1989) descreveu seu modelo como sendo baseado em aspectos cognitivos da busca de informação, caracterizado por conter categorias gerais, independentes, ou seja, não são definidas como fases de um processo. Como sinalizado anteriormente, os modelos de comportamento de busca e uso de informação são sempre elaborados a partir de estudos com grupos específicos de usuários. No caso de Ellis, ele estudou grupos de cientistas sociais de vários departamentos da Universidade de Sheffield e considera seis atividades básicas do usuário durante a situação de busca por informação:

1) inicialização - o usuário começa a buscar pela informação. Inicia-se com uma visão geral do tema a ser estudado e pode indicar desdobramentos para o aprofundamento ulterior a busca. No caso de usuários de informação científica, por exemplo, essa etapa se refere ao levantamento bibliográfico, consultas a fontes secundárias de informação, entre outras fases iniciais; para usuários não especialistas, pode-se considerar como etapa inicial um telefonema a um amigo, conversar com parentes e colegas, entre outras.

2) encadeamento - o usuário busca informações em fontes indicadas pelas primeiras fontes que encontrou, fazendo ligações e relações entre fontes, buscando sempre encontrar algo mais a respeito da informação procurada. 
3) navegação - o usuário realiza busca semiestruturada em fontes potenciais, num processo semelhante ao browsing, conforme descrito por Naves (1998): uma atividade não orientada, não programada, informal, não sistemática, casual, ou seja, é quando, por exemplo, o usuário apenas caminha por entre as estantes do acervo olhando as lombadas dos livros, folheando-os, observando catálogos, sem uma análise mais profunda dos documentos.

4) diferenciação - o usuário filtra e seleciona as fontes de informação que o interessam. Por meio da diferenciação, o usuário avalia o tipo de conteúdo e decide pela relevância ou não do material.

5) monitoramento - o usuário verifica por atualizações nas fontes de informação de seu interesse, compreendendo, por exemplo, o acompanhamento das publicações em determinado tipo de periódico ou do desenvolvimento de um determinado campo do saber. Atualmente, existem vários bancos de dados que permitem que o usuário crie e gerencie perfis de interesse, através dos quais o sistema avisa ao usuário, por e-mail, quando da inserção de novos materiais potencialmente relevantes.

6) extração - o usuário utiliza sistematicamente as fontes de informação tidas por ele como essenciais. Compreende exatamente a etapa do uso da informação e é considerada a atividade mais focada e direta, na qual o usuário tende a permanecer mais tempo.

\section{Contribuiçóes posteriores ao Modelo de Ellis}

Conforme supramencionamos, Ellis (1989) desenvolveu seu modelo de comportamento de busca e uso da informação a partir do estudo do comportamento de cientistas socais. Quatro anos mais tarde, em 1993, o modelo original de Ellis foi ampliado por Ellis, Cox e Hall. O propósito desse novo estudo foi criar um modelo de comportamento de busca de informação de acadêmicos da área de Física e Química, utilizando um método semelhante ao que havia sido usado por Ellis em 1989 para o estudo de cientistas socais. Como resultado, Ellis, Cox e Hall (1993) propuseram a ampliação do modelo com a inserção de duas novas cetegorias, passando ele a constar de oito e não mais de seis atividades:

7) verificação - o usuário confere a veracidade das informações encontradas. Requer o cuidado de identificar possíveis erros ou inconsistência na informação, checar a confiabilidade da fonte, a completeza e a atualidade da informação. 
8) finalização - o usuário volta a buscar informação ao final de um projeto. Por exemplo, durante a preparação de artigos para publicação, quando o cientista retorna à literatura, com a intenção de verificar se não há algum trabalho muito similar ao seu, ou de outro que poderia alterar os rumos da sua pesquisa.

Tanto o modelo de Ellis (1989) quanto o proposto por Ellis, Cox e Hall (1993) definiram o modelo não composto por etapas que se sucedem, mas por atividades numa ordem que pode variar. Isso porque o usuário pode repetir alguma ou algumas dessas atividades ou entrar no processo de busca por informação partindo de qualquer uma das oito atividades.

Em 2005, por ocasião de sua pesquisa de mestrado, Crespo utilizou o modelo de comportamento de busca e uso de informação de Ellis, Cox e Hall (1993) para aplicá-lo a pesquisadores da área de Biologia Molecular e Biotecnologia, com o objetivo de verificar se seu comportamento de busca é afetado pela informação científica disponível em meio digital, especificamente pelo periódico científico eletrônico. Como um dos resultados de sua pesquisa, a autora propôs mais uma categoria a ser agregada ao modelo de Ellis:

9) personalização ${ }^{2}$ - o usuário personaliza os recursos de busca nos sistemas de recuperação de informações. Essa categoria se refere aos recursos e funcionalidades que o navegador de internet ou o próprio layout do banco de dados oferece, como por exemplo, assinalar itens de interesse, adicionar elementos às páginas web, salvar preferências de navegação, adicionar uma página aos favoritos, alterar o tamanho de fonte, entre outras possibilidades de personalização.

Barros (2008), por sua vez, estudando o comportamento de busca de informação dos usuários do Arquivo Público do Maranhão, analisaram, à luz do modelo de comportamento de busca de informação de Ellis, Cox e Hall (1993), se as estratégias metacognitivas do profissional da informação do arquivo supramencionado são semelhantes ou diferentes das traçadas pelos pesquisadores no comportamento de busca da informação. Dentre outros resultados da pesquisa, elas descobriram uma nova categoria a ser incorporada ao modelo:

2 Dispensamos a discussão sobre a adequação do termo personalização em vez de customização, por julgar que a explanação de Isabel Crespo não deixa dúvidas sobre a que realmente ela se refere. Uma discussão sobre a controvérsia envolvendo esses conceitos está presente em Santos (2002). 
10) transcrição - o usuário transcreve dados e informações. No entanto, Barros (2008, p. 135) alertam para o fato de que essa nova categoria é própria da pesquisa em arquivos permanentes: "Por existirem neles manuscritos, é necessário o conhecimento da Paleografia para que imprima maior fidedignidade possível ao teor do documento" e assinalam um fator condicionante: "Na realidade, a inserção da transcrição como categoria no modelo de busca de informação de Ellis, Cox e Hall (1993), só é possível aos Arquivos Históricos pela própria peculiaridade da constituição de conjuntos documentais por manuscritos".

\section{Estudo empírico junto às dissertaçóes e teses defendidas em Programas de Pós-Graduação no Brasil e proposta de inserção de mais uma categoria ao modelo de Ellis}

É importante frisar que os autores das vinte e duas dissertações e quatro teses que compuseram o corpus documental da presente pesquisa realizaram suas investigações tendo como sujeitos grupos sociais bastante variados, como por exemplo: sindicalistas, funcionários de indústrias e de fábricas, alunos participantes de empresas juniores, empresários, pacientes da área da saúde, entre outros. Essa variedade traz a vantagem de termos um quadro bastante amplo, plural, o que favorece o conhecimento generalista do modo como os usuários buscam e usam informação na atualidade.

Naturalmente, a análise do material trouxe à tona questões referentes, por exemplo, a fatores que motivam a busca por informação, à preferência por um determinado tipo de mídia, ao tempo empregado para a leitura, a preferências de busca e ao tipo de uso que é feito da informação recuperada. Todos esses temas representam, contudo, questões secundárias a este artigo, motivo pelo qual não nos deteremos na análise de todos eles neste momento.

O que nos chamou atenção, no entanto, diz respeito unicamente ao fator uso da informação, exatamente porque verificamos um tipo de uso não contemplado no modelo de Ellis (1989) nem no de Ellis, Cox e Hall (1993), mas que se mostrou frequente (em torno de $70 \%$ ) nos grupos sociais estudados e descritos nas conclusões das dissertações e teses por nós analisadas, conforme exemplificamos com os trechos abaixo:

- há valorização da possibilidade de que o conhecimento adquirido seja ampliado e reproduzido, para ajudar aos outros pacientes. (Usuários de informação da área da saúde);

- os próprios usuários são também fontes de informação, por meio do 
compartilhamento de seus conhecimentos, experiências cotidianas e fontes de informação conhecidas e em que confiam. (Usuários de informação disponível na web);

- os profissionais afirmam compartilhar informações entre os colegas de equipe, embora, para a maioria, não exista espaço físico, nem a organização possua política de incentivo a esse compartilhamento. (Funcionários de empresas de informática).

Assim, tendo identificado e analisado essas regularidades existentes no que diz respeito aos principais resultados das dissertações e teses selecionadas para compor o corpus documental deste artigo, identificamos a necessidade de também propor uma ampliação ao modelo clássico de Ellis. Percebeu-se que os sujeitos das pesquisas, os usuários da informação, não buscam informação pensando exclusivamente em resolver problemas e satisfazer necessidades pessoais, havendo uma forte propensão à solidarização com outras pessoas menos informadas, o que as leva a procurar informação para distribuir. Assim, propomos uma décima primeira categoria ao modelo de comportamento de busca e uso de informação de Ellis:

11) compartilhamento - o usuário compartilha a informação recuperada. Entenda-se por compartilhamento a divulgação da informação de maneira ampla, como aquela que é utilizada em treinamentos de empregados, para lecionar a estudantes, repassar a amigos e parentes, veicular em meios de comunicação, postar em redes sociais na internet, dentre outras possibilidades.

\section{Conclusão}

O homem, ser social e cognitivo, está em constante transformação do seu eu, enquanto contribui para a transformação do seu entorno, socialmente, culturalmente e tecnologicamente. Essa dinamicidade se reflete nos modos como consumimos informação, principalmente após a popularização da internet.

Naturalmente, nas últimas décadas, mudaram as formas como buscamos e acessamos a informação de que precisamos, razão pela qual o profissional da informação não deve negligenciar estudos e pesquisas com foco no comportamento do usuário, através dos quais poderão ser planejados e ofertados produtos e serviços de acordo com as reais necessidades dos usuários, já que as necessidades mudam, assim como os comportamentos de acesso e uso informacional. 
Exemplo disso são as categorias presentes no modelo de comportamento de busca e uso de informação elaborado por Ellis em 1989: inicialização, encadeamento, navegação, diferenciação, monitoramento e extração. Já em 1993, houve a inserção de mais duas categorias: verificação e finalização. Em 2005, mais uma ampliação foi proposta, pelo que se agregou a categoria personalização. Em 2011, outra categoria foi descoberta, a transcrição. Agora, mais de vinte anos depois da elaboração do modelo original, encontramos a necessidade de nova ampliação e lançamos a proposta de inserção da categoria Compartilhamento.

É fácil perceber que o compartilhamento de informações aparece aqui muito ligado às mídias eletrônicas e digitais, às possibilidades de interação e colaboração oferecidas pela web 2.0, se considerarmos que o compartilhamento do qual falamos diz mais respeito à disseminação de informações com o auxílio de um computador conectado à internet e menos ao fato de "espalharmos" informação boca a boca entre familiares e amigos, ou em salas de aula, por exemplo.

No entanto, isso nos mostra a força e abrangência que as tecnologias da informação e da comunicação têm em nossa sociedade, tão apaixonada por artefatos e inovações tecnológicas que nos deixem conectados ao mundo em tempo real e o tempo todo. Prova disso é que vemos cada vez mais pessoas de todas as idades portando notebooks, tablets e smartphones com acesso à rede mundial de computadores, para se manterem informadas e para informarem, para se comunicarem e para compartilhar. Estaríamos então, partindo para uma sociedade que vive um novo regime de informação, o da informação compartilhada? Bem, muitos tentaram nomear o nosso tempo: sociedade da informação, sociedade do conhecimento, sociedade em rede... Por que não pensar, então, em uma sociedade do compartilhamento?

\section{REFERÊNCIAS}

Barros, D. S. (2008), Dimensões metacognitivas no comportamento de busca de informação: Estudo de usuário no Arquivo Público do Estado do Maranhão (APEM). 157 f. Dissertação (Mestrado em Ciência da Informação)-Programa de Pós-Graduação em Ciência da Informação, Universidade Federal da Paraíba, João Pessoa.

Belkin, N. J. (1980), "Anomalous states of knowledge as a basis for information retrieval", The Canadian Journal of Information Science, n. 5, p. 133-143.

Capurro, R. (2007), "Epistemología y ciencia de la información", Enl@ce, Venezuela, v. 4, n. 1, p. 11-29. 
Cruz, R. (2011), Redes sociais virtuais de informação sobre amor: comportamento e cultura informacional de usuários do Orkut. $324 \mathrm{f}$. Dissertação (Mestrado em Ciência da Informação)-Universidade Federal de Minas Gerais.

Crespo, I. M. (2005), Um estudo sobre o comportamento de busca e uso de informação de pesquisadores das áreas de biologia molecular $e$ biotecnologia: impactos do periódico científico eletrônico. $121 \mathrm{f}$. Dissertação (Mestrado em Comunicação e Informação)-Universidade Federal do Rio Grande do Sul, Porto Alegre.

Dervin, B. (1983), "From the mind's eye of the user?: the sense -making qualitative-quantitative methodology", in J. D. Glazier e R. R. Powell, Qualitative research in information management, Englewood: Libraries Unlimited, p. 52-64. Disponível em: http:// comminfo.rutgers.edu/ tefko/Courses/612/Articles/DervinMind seye.pdf Acesso em: 18 mar. 2013.

Ellis, D. (1989), "A behavioral model for information retrieval system design", Journal of Information Science, Cambridge, v. 15, n. 4-5, p. 237-247.

-; Cox, D.; Hall, K. (1993), "A comparison of the information seeking patterns of researchers in the physical and social sciences", Journal of Documentation, London, v. 49, n. 4, p. 356-369.

Kuhlthau, C. C. (1991), "Inside the search process: information seeking from the user's perspective", Journal of the American Society for Information Science, v. 42, n. 5, p. 361-371. Disponível em: http: //comminfo.rutgers.edu/ belkin/612-05/kuhlthau-jasist-91.pdf Acesso em: 18 mar. 2013.

Laland, K. N.; Brown, G. R. (2002), Sense and nonsense: evolutionary perspectives on buman behaviour, New York: Oxford University Press.

Mesquita, R.; Duarte, F. (1996), Dicionário de Psicologia, [S.1.]: Plátano. Disponível em: http://xa.yimg.com/kq/groups/24695084/646 305773/name/Dicion\%C3\%A1rio +de+Psicologia+-+Raul+Mes quita+e+Fernanda+Duarte.pdf Acesso em: 26 mar. 2013.

Milne, P. (1999), "Electronic access to information and its impact on scholarly communication", in The Ninth Australasian Information Online \& On Disc Conference And Exhibition, 1999, Sydney. Proceedings... Sydney: [s.n.]. Disponível em: http://www.csu. edu.au/special/online99/proceedings99/305b.htm Acesso em: 28 abr. 2013.

Naves, M. M. L. (1998), "Aspectos conceituais do browsing na recuperação da informação", Ciência da Informação, Brasilia, v. 27, n. 3, set.

Pinheiro, L. V. R. (1982), Usuários-Informação: o contexto da ciência e da tecnologia, Rio de Janeiro: LTC - Livros Técnicos e Científicos, CNPq/IBICT.

Santos, A. L. R. (2002), Informação fast-food: um estudo de caso do jornal Último Segundo do Portal IG. Dissertação (Mestrado)-Universidade Federal da Bahia, Salvador. 
Wilson, T. D. (2000), "Human information behavior", Informing Science: the International Journal of an Emerging Transdiscipline, Special Issue on Information Science Research, v. 3, n. 2, p. 49-55. (1981), "On user studies and information needs", Journal of Documentation, v. 37, n. 1, p. 3-15.

-; Walsh, C. (1996), "Information behaviour: an interdisciplinary perspective", British Library Research and Innovation Report, n. 10. Disponível em: http://informationr.net/tdw/publ/infbehav/ cont.html Acesso em: 18 mar. 2013 